\title{
Boron Doping of Microcrystalline and Nanocrystalline Diamond Films: Where is the Boron Going?
}

Paul William May ${ }^{1}$, William J Ludlow ${ }^{1}$, Matthew Hannaway ${ }^{1}$, James A Smith ${ }^{1}$, Keith N Rosser ${ }^{1}$, and Peter J Heard ${ }^{2}$

${ }^{1}$ School of Chemistry, University of Bristol, Cantock's Close, Bristol, BS8 1TS, United Kingdom

${ }^{2}$ Interface Analysis Centre, University of Bristol, 121 St. Michael's Hill, Bristol, BS2 8BS, United Kingdom

\section{ABSTRACT}

We present data showing how the electrical conductivity and Raman spectra of boron doped 'cauliflower'-type nanocrystalline (c-NCD) CVD diamond films vary as a function of B content. The conductivity is roughly linear as a function of $\mathrm{B}$ content between an onset threshold of $\sim 5 \times 10^{20} \mathrm{~cm}^{-3}$ up to $\sim 6 \times 10^{21} \mathrm{~cm}^{-3}$, with the higher concentrations giving near metallic conductivity values. The onset threshold may be due to compensating donors due to the large number of impurities and defects in these films. The position of the Lorentzian contribution to the $500 \mathrm{~cm}^{-1}$ Raman feature was used to estimate the B content and compared to the value measured using SIMS. We found that the Raman method overestimated the concentration of B by a factor of $\sim 5$ for these c-NCD films. The shortfall may be explained if only a small fraction of the B found in the small-grained films is being incorporated into substitutional sites. We conclude that in diamond films with a high concentration of grain boundaries, the majority of the B ( $80 \%$ in some cases) must be present at or in the grain boundaries.

\section{INTRODUCTION}

Diamond films produced by chemical vapour deposition (CVD) can be doped with boron to produce a p-type semiconducting material with electrical conductivity that ranges from insulating to metallic, depending upon the doping level [1]. The boron dopant atoms act as electron acceptors, and form a band located $\sim 0.35 \mathrm{eV}$ above the valence band edge. At low temperatures or at boron concentrations $<10^{17} \mathrm{~cm}^{-3}$ conduction occurs through holes in the valence band contributed by ionised substitutional $\mathrm{B}$. At higher doping levels, conduction occurs by nearest-neighbour and variable range hopping of holes between ionised B sites [2], accompanied by a drop in mobility [3]. At very high doping levels, an impurity band is formed, giving rise to metal-like conductivity.

A complication is that polycrystalline boron-doped CVD diamond films possess grain boundaries containing a small-volume fraction of non-diamond carbon impurities. Consequently, the electrical conductivity of the film is a complicated function of the combined effects of the boron-doping level, the grain boundaries, and the impurities. As the grain size in the films becomes smaller, i.e. from microcrystalline diamond (MCD) to nanocrystalline diamond (NCD), the relative importance of these grain boundaries increases. As a result, most reports concerning B-doped diamond, both fundamental science-based and applications-led, have concentrated upon epitaxially-grown diamond or MCD films. There have been relatively few 
studies concerning B-doped NCD films, with the majority concerning their applications for electrochemical electrodes [4] or other devices [5,6]. However, a recent theoretical study by Barnard and Sternberg [7] predicted that boron is likely to be positioned at the surface of isolated nanoparticles and at the grain boundaries in NCD films.

Unfortunately there is no rigorous definition for NCD in the literature, and the only agreed property is that the films have grain sizes in the nm range, typically between 10-500 nm, although even the exact range is arguable. In fact, a number of films with quite distinct characteristics all come under the broad umbrella of NCD [8]. One type of NCD film often contains a significant fraction of non-diamond material, and exhibits no faceting or evidence of columnar growth, even at thicknesses of several $\mu \mathrm{m}$. Some of these NCD films have a rounded appearance and as a result, their morphology is often referred to as 'ballas' (meaning ball-like) or cauliflower-like $[9,10]$. We shall call these types of films 'cauliflower NCD' (c-NCD). Thicker c-NCD films can be grown with essentially flat surfaces with nm smoothness, and this allows them to be patterned with much higher resolution than MCD. Decreasing the diamond crystallite size below $10 \mathrm{~nm}$ produces what has come to be known as 'ultrananocrystalline' diamond (UNCD) films [11]. However, there are as yet no reports of successful B-doping of UNCD [12]. In this paper we shall concentrate upon comparing the doping behaviour, electrical properties, and Raman characteristics of boron-doped c-NCD films.

Studies of the Raman spectra from B-doped diamond films have also been restricted almost exclusively to epitaxial or MCD films [13]. The diamond phonon observed at $1332 \mathrm{~cm}^{-1}$ is a symmetric Lorentzian at low boron concentration, but as the boron concentration increases above a threshold of $\sim 10^{20} \mathrm{~cm}^{-3}$ - corresponding to the onset of metallic conductivity - there is an abrupt and pronounced change toward an asymmetric Fano-like lineshape [14]. This asymmetry is caused by a quantum mechanical interference between the zone-centre Ramanactive optical phonon and the continuum of electronic states induced by the presence of the dopant. The threshold for the appearance of the Fano-like lineshape was found to depend on the excitation laser wavelength [15]. The Raman peak also shifts to lower wavenumber with increased boron concentration, and is accompanied by a wide signal $\left(300-1330 \mathrm{~cm}^{-1}\right)$ with structures around 500 and $1225 \mathrm{~cm}^{-1}$ (although these structures do not appear when using ultraviolet $\left(244 \mathrm{~cm}^{-1}\right)$ excitation [16]). Such results were first reported for epitaxially-grown diamond [17,18] and then confirmed [14,19] for polycrystalline boron-doped films. Wang et al. [16] found that this shift in the peak position was also a function of excitation wavelength in the range $244-514 \mathrm{~nm}$. The origin of the 500 and $1225 \mathrm{~cm}^{-1}$ peaks is uncertain, but their positions approximately agree with two maxima in the phonon density of states (PDOS). They may therefore be connected with a relaxation of the wavevector selection rules, and if so, they may well be associated with the actual boron incorporation in the lattice, rather than the hole concentration [20]. There is also evidence [21] that the $500 \mathrm{~cm}^{-1}$ peak originates from local vibrational modes of boron pairs [22], which cause some distortion in the diamond lattice around these isolated defects.

For very heavy B-doping, the Fano parameters saturate and it becomes difficult to discern the position of the zone-centre phonon, with the peaks at 500 and $1225 \mathrm{~cm}^{-1}$ dominating the spectrum. For these conditions, the $500 \mathrm{~cm}^{-1}$ peak can be fitted with a combination of Gaussian and Lorentzian line shapes. The wavenumber position, $\omega$, of the Lorentzian component approximately obeys [20]:

$$
[\mathrm{B}] / \mathrm{cm}^{-3}=8.44 \times 10^{30} \exp (-0.048 \omega)
$$

where $\omega$ is in $\mathrm{cm}^{-1}$ and the boron content, [B], is in the range from $2 \times 10^{20}$ to $1 \times 10^{22} \mathrm{~cm}^{-3}$.

Here, we present results for the Raman spectra of boron-doped c-NCD films as a function of $\mathrm{B}$ content, using a range of excitation wavelengths from the ultraviolet to the infrared. 


\section{EXPERIMENT}

The diamond film were all grown in a standard hot filament CVD reactor using $5 \% \mathrm{CH}_{4} / \mathrm{H}_{2}$ process gases at a pressure of 20 Torr. The substrate was undoped single crystal (100) Si, abraded ultrasonically before deposition using a slurry of $100 \mathrm{~nm}$ diamond grit in water. Rhenium was used for the filament material rather than tantalum since Re does not act as a sink for carbon or boron species, unlike Ta which can absorb significant amounts of B and C for many hours into the deposition run [23]. The filament temperature was kept constant at $2400^{\circ} \mathrm{C}$ and monitored using a 2-colour optical pyrometer, and the substrate temperature was maintained at $\sim 900^{\circ} \mathrm{C}$. Diborane $\left(\mathrm{B}_{2} \mathrm{H}_{6}\right)$ gas diluted in $\mathrm{H}_{2}$ was used as the source of $\mathrm{B}$ at concentrations between 10-12000 ppm with respect to $\mathrm{CH}_{4}$. The c-NCD films were grown for $8 \mathrm{~h}$ giving films $\sim 4 \mu \mathrm{m}$ thick, and the film morphology can be seen in Fig.1. For comparison, an undoped film was grown in a different but otherwise identical HF CVD reactor that had never used boron-containing gases.

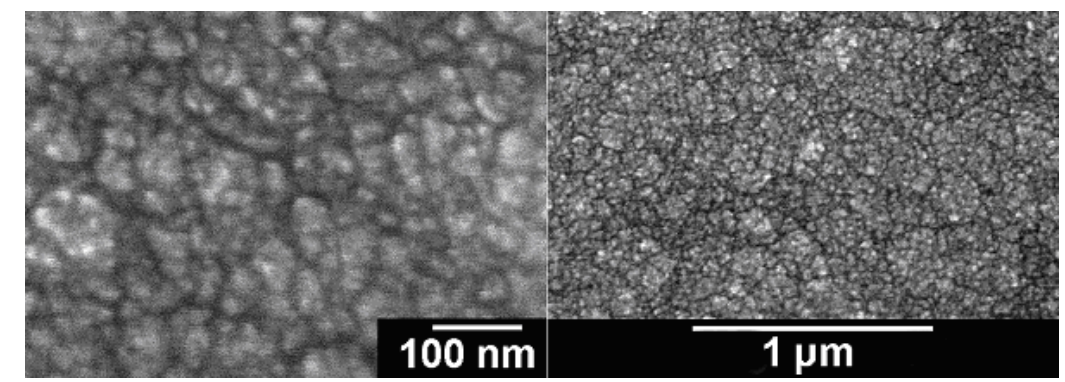

Figure 1. Scanning electron micrographs of the surface of c-NCD film with grains sizes 20-100 nm.

Electrical conductivity measurements were made by a four-point probe method in which spring-loaded probes were pressed into contact with the surface of the film and the resistance measured over a distance of $\sim 5 \mathrm{~mm}$. Note that even though the four-point probe measurements supposedly remove most of the effects of contact resistance, these measurements were of the effective surface conductivity, and might not correspond to the bulk conductivity. Linear plots of current against applied voltage, passing through the origin, were obtained, showing that the contacts were Ohmic.

Boron content within the film was measured using secondary ion mass spectrometry (SIMS), with the B:C count ratio being calibrated with respect to those from two reference samples. The first reference was a single crystal diamond implanted with a known dosage of B, and therefore containing a known B-content. The second reference was an undoped CVD diamond film with zero B content, providing the background count level for B.

Laser Raman spectra were obtained at room temperature using a Renishaw 2000 spectrometer and a range of excitation wavelengths and lasers: UV $325 \mathrm{~nm}(\mathrm{HeCd})$, blue $488 \mathrm{~nm}$ $\left(\mathrm{Ar}^{+}\right)$, green $514 \mathrm{~nm}\left(\mathrm{Ar}^{+}\right)$, red $625 \mathrm{~nm}$ HeNe, IR $785 \mathrm{~nm}$ (IR diode), and far-IR $830 \mathrm{~nm}$ (IRdiode). Undoped or lightly doped films often exhibited a large rising or falling photoluminescent (PL) background upon which the much smaller Raman features would sit. This PL background 
would decrease markedly with even trace amounts of B doping, and for B contents $>5 \times 10^{18} \mathrm{~cm}^{-3}$ it disappeared altogether giving a flat baseline. In order to see the small Raman features more clearly, and to reproduce the spectra from the lightly doped films on the same scale as the heavily doped ones, it was useful to subtract this PL background where necessary. This was done by fitting a polynomial curve to the background, and then subtracting this to obtain a processed spectrum. With increasing B content, the films became visibly darker and often looked bluish, and the Raman signals became correspondingly less intense. Longer accumulation times were required to obtain reasonable signal:noise levels in these samples.

In those samples where the $500 \mathrm{~cm}^{-1}$ band was discernible (i.e. the more heavily doped samples), the band was fitted to a Gaussian curve centred $\sim 500-550 \mathrm{~cm}^{-1}$ and a Lorentzian curve centred $\sim 460-505 \mathrm{~cm}^{-1}$ following the procedure mentioned in the Introduction and outlined in ref.[20]. Using the relationship between the B content and the wavenumber of the Lorentzian component of the $500 \mathrm{~cm}^{-1}$ band (Eqn.1), it was possible to estimate the doping levels in these films, with which to compare the values from SIMS.

Table 1. B content as measured by SIMS, and as estimated from fitting the $500 \mathrm{~cm}^{-1}$ Raman peak using Eqn.(1), plus 4-point resistance measurements for the c-NCD films.

\begin{tabular}{|c|c|c|c|}
\hline Film & $\begin{array}{c}\text { B content (SIMS) } \\
/ \mathbf{~ c m}^{-3}\end{array}$ & $\begin{array}{c}\text { B content } \\
\text { from Eqn.(1) } \\
/ \mathbf{~ c m}^{-3}\end{array}$ & $\begin{array}{c}\text { 4-point } \\
\text { resistance } / \boldsymbol{\Omega}\end{array}$ \\
\hline C1 & 0 & - & $1 \times 10^{7}$ \\
\hline C2 & $6.0 \times 10^{18}$ & - & $5 \times 10^{6}$ \\
\hline C3 & $6.1 \times 10^{20}$ & - & 6480 \\
\hline C4 & $1.1 \times 10^{21}$ & $3.4 \times 10^{20}$ & 294 \\
\hline C5 & $2.9 \times 10^{21}$ & $4.5 \times 10^{20}$ & 66 \\
\hline C6 & $6.1 \times 10^{21}$ & $9.6 \times 10^{20}$ & 43 \\
\hline
\end{tabular}

\section{RESULTS}

\section{$\underline{\text { 1. Electrical results }}$}

The doping levels and resistance values for the films are given in Table 1. The values of the boron content in the films are plotted against 4-point conductance (=1/resistance) in Fig. 2 . The trend is roughly linear, however the conductivity is much less than has been previously observed for B-doped MCD films for the same B content [24,25]. This suggests that the doping efficiency for c-NCD films is greater than for MCD, and this might be explained if a lower proportion of the incorporated $\mathrm{B}$ atoms were contributing to doping in $\mathrm{c}-\mathrm{NCD}$. The reduced doping efficiency suggests that a significant amount of the B is being incorporated in positions (such as at the grain boundaries), which do not improve the conductivity to the same extent as doping the crystallites themselves. Also, there appears to be a threshold for B doping of $\sim 5 \times 10^{20} \mathrm{~cm}^{-3}$, below which the conductance is almost zero. This may be a result of the large number of defects and impurities in these films which can compensate for the boron and suppress the conductivity at low doping levels. 


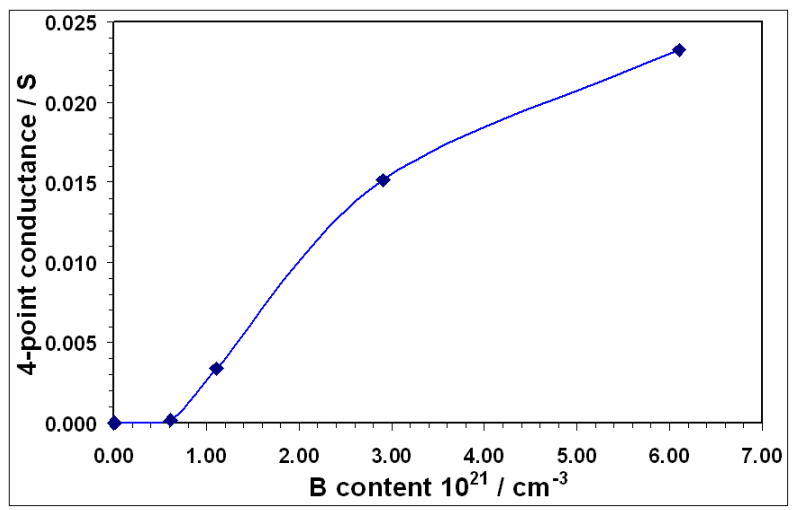

Figure 2. B content as measured by SIMS plotted against measured 4-point conductance (1/resistance) for the c-NCD types of films. B content values are estimated to be accurate to $\pm 10 \%$.

\section{Laser Raman spectra}

Although laser Raman spectra were obtained for 6 different laser wavelengths, only those spectra which show substantial differences from each other will be presented here.

The Raman spectra of the c-NCD films excited in the UV, green and IR are shown in Figs.3, 4 and 5. The UV spectra (Fig.3) show a large $\mathrm{G}$ band, and a much reduced diamond peak. The $1170 \mathrm{~cm}^{-1}$ peak is also relatively prominent, which indicates that the amount of non-diamond carbon present in these c-NCD films is significantly high, which is consistent with their small grain size and high grain boundary density. Notably, the $1225 \mathrm{~cm}^{-1}$ peak is absent from the spectrum of even the most highly doped sample, as reported previously for epitaxial diamond [16]. With increasing B content, the diamond peak and the $1170 \mathrm{~cm}^{-1}$ peak reduce in intensity compared to the broad D and $\mathrm{G}$ bands, possibly indicating that the crystallite size is decreasing (although this couldn't be discerned by SEM analysis) and that the films are becoming dominated by amorphous, rather than crystalline material. Indeed, the spectrum for sample C6 more closely resembles that from a diamondlike carbon film than a diamond film.

The green Raman spectra all exhibited extremely strong PL backgrounds, which have been subtracted in Fig.4 to allow comparison between the important features of the spectra. The diamond peak is again less prominent, whereas the $1170 \mathrm{~cm}^{-1}$ peak is now relatively intense and its companion peak $\left(\sim 1450 \mathrm{~cm}^{-1}\right)$ is now stronger than the $\mathrm{D}$ and $\mathrm{G}$ bands. For higher $\mathrm{B}$ concentrations, the $\mathrm{G}$ band becomes more structured, with two peaks appearing at 1560 and $1600 \mathrm{~cm}^{-1}$. With increasing B content, the $1225 \mathrm{~cm}^{-1}$ peak intensity increases, eventually hiding the weaker $1170 \mathrm{~cm}^{-1}$ and diamond peaks. The diamond peak itself becomes wider and smaller with increasing B content. The Si peak (from the substrate) also decreases with B content, until it, too, is hidden by the more intense $500 \mathrm{~cm}^{-1}$ band. 


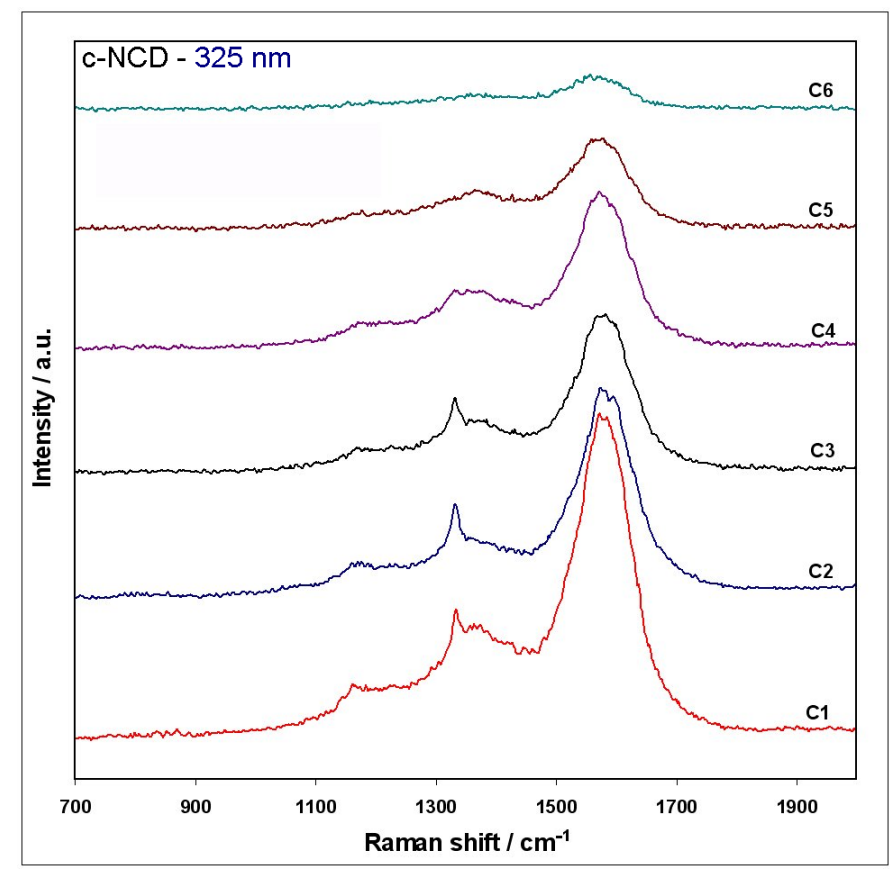

Figure 3. Raman spectra for the different boron-doped c-NCD films using UV (325 nm) excitation. The films increase in B content from C1 (undoped) to C6 heavily doped, with the details given in Table 1. The spectra have been offset vertically from each other for clarity, with no PL background subtraction.

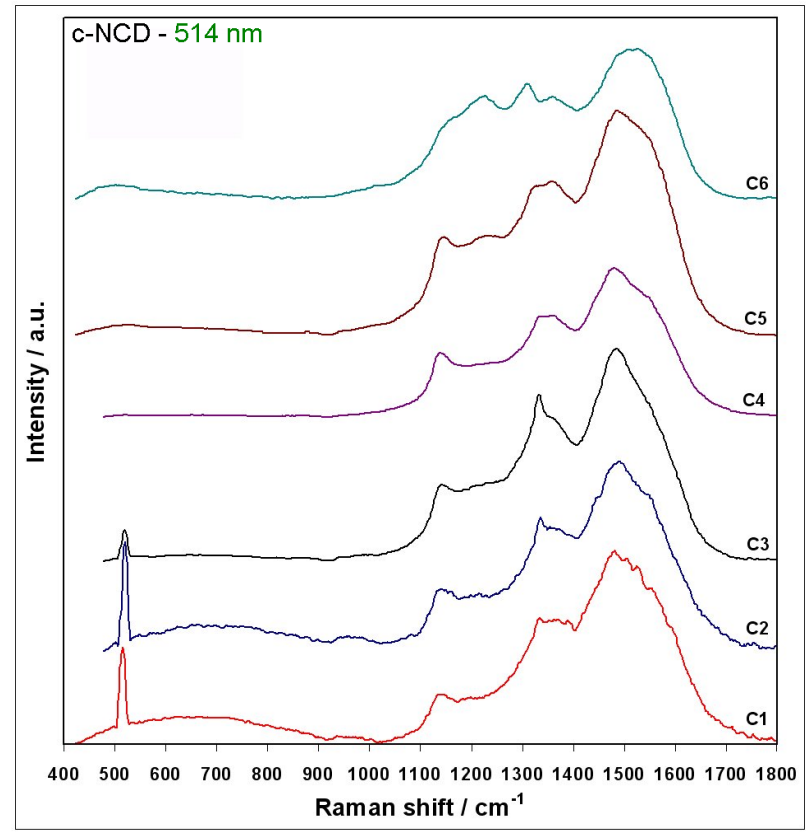

Figure 4. Raman spectra for the different B-doped c-NCD films using green $(514 \mathrm{~nm})$ excitation. The spectra have been offset vertically from each other for clarity, with PL background subtraction applied to all of them to produce a flat baseline. The PL background levels for $\mathrm{C} 1$ and $\mathrm{C} 2$ were respectively $\sim 10 \times$ and $\sim 5 \times$ those of the more heavily doped films, and uncertainties in the background subtraction procedure may have artificially produced the broad features seen between $400-900 \mathrm{~cm}^{-1}$ in the spectra of $\mathrm{C} 1$ and $\mathrm{C} 2$. 
The IR spectra (Fig.5) show very few features for low B content films, with just a Si peak and a broad PL background centred around $1330 \mathrm{~cm}^{-1}$. However, with increasing B, the PL background diminishes allowing more features, such as the diamond line, the $1170 \mathrm{~cm}^{-1}$ peak, and the $\mathrm{G}$ band to become apparent. With further increases in $\mathrm{B}$, the $1225 \mathrm{~cm}^{-1}$ peak grows in intensity and shifts to higher wavenumber, while the diamond line attains the Fano lineshape. At the same time, the Si line disappears as the $500 \mathrm{~cm}^{-1}$ feature becomes much more intense.

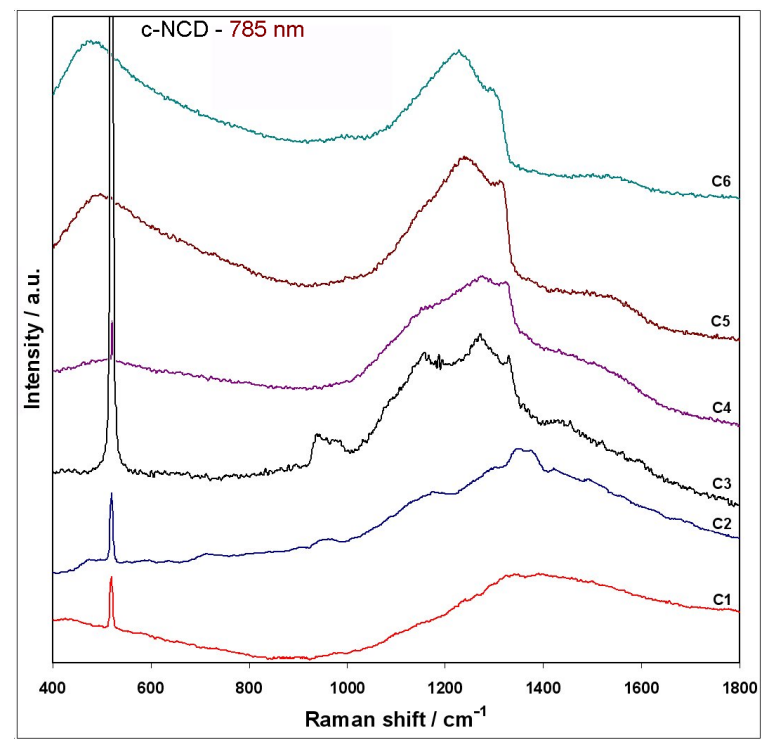

Figure 5. Raman spectra for the different B-doped c-NCD films using IR $(785 \mathrm{~nm})$ excitation. The spectra have been offset vertically from each other for clarity, with no PL background subtraction. The intensities in the spectra for $\mathrm{C} 1$ and $\mathrm{C} 2$ have been scaled down by a factor of 10 to facilitate comparison between spectra. The large feature around $900-1000 \mathrm{~cm}^{-1}$ is the second order from the Si line.

Table 1 shows the values for B concentration for the heavier doped films estimated from the Raman fit of the $500 \mathrm{~cm}^{-1}$ peak and using Eqn.(1). The calculated values underestimate those from SIMS by factors of between 3 and 6. This is in contrast to values for MCD films which are all within a factor of 2 of those measured by SIMS [24]. The difference arises since SIMS counts all the B present in the film, whereas the Raman method only counts those boron atoms which directly affect the $500 \mathrm{~cm}^{-1}$ band.

\section{CONCLUSIONS}

We have presented data showing how the conductivity and Raman spectra of B doped c-NCD films vary as a function of B content. We found that the conductance of B-doped c-NCD films was much lower than those for the larger grained MCD films, for the same B content. We also found that Eqn.(1) overestimates the concentration of B in the films by a factor of $\sim 5$ for c-NCD films, although it remains reasonably accurate for MCD films. Eqn.(1) measures only those boron atoms which directly incorporate into the diamond lattice (and hence affect the position of the $500 \mathrm{~cm}^{-1}$ band). The shortfall between these values and the 'true' values 
measured by SIMS may be explained if only a small fraction of the B found in the small-grained films is being incorporated into substitutional sites. The majority of the B ( $80 \%$ in some cases) must be present at sites that do not contribute to the continuum of electronic states that give rise to metallic conductivity and the Fano effects. Such sites may include (a) interstitials, (b) the surface of the crystallites, or (c) bonded within the non-diamond carbon impurities present at the grain boundaries. This is consistent with the theoretical predictions [7] that B is likely to be preferentially located in or near grain boundaries. This suggests that heavy doping of nanograined diamond films will give rise to a material with many different conducting regions, and possibly different conducting pathways and mechanisms. Not only are the nanograins doped, possibly up to the point of metallic conductivity, but the surface layer of each grain may have a higher concentration of $\mathrm{B}$, allowing conducting pathways around each grain.

Furthermore, the amount of non-diamond carbon at the grain boundaries, specifically graphite, increases with B addition, giving yet another potential conduction route. And finally, the presence of B inside this graphitic impurity layer may provide yet another mechanism for electron transport. Some of these ideas will be explored in a later paper. Nevertheless, despite the complexity of the underlying conduction mechanisms present in c-NCD films, they can be fabricated with controllable conductivities and nm-smooth surfaces, which might make them ideal candidates for use as an electronic material.

\section{ACKNOWLEDGMENTS}

The authors would like to thank Jacob Filik, Martin Kubal \& Tim Batten, and Keith Hallam \& Diana Edwards for use of their far IR, blue, and red Raman systems, respectively, Jonathan Jones for the high resolution SEM photos.

\section{REFERENCES}

1. R. Kalish, Carbon, 37 (1999) 781.

2. B. Massarani, J.C. Bourgoin, and R.M. Chrenko, Phys. Rev. B 17, (1978) 1758.

3. K. Nishimura, K. Das, and J. T. Glass, J. Appl. Phys. 69, (1991) 3142.

4. Yu. V. Pleskov, Russ. J. Electrochem. 38 (2002) 1275.

5. M. Willander, M. Friesel, Q.-ul. Wahab and B. Straumal, J. Mater. Sci: Maters in Electron. 17 (2006) 1.

6. D.L. Dreifus, A. Collins, T. Humphreys, K. Das, P.E. Pehrsson, eds, Diamond for Electronic Applications, MRS Symp. Proc. Vol 416 (Materials Research Society, Pittsburgh, 1996).

7. A.S. Barnard and M. Strernberg, J. Phys. Chem. B 110 (2006) 19307.

8. P.W. May, Chemical vapour deposition - A route to microcrystalline, nanocrystalline, ultrananocrystalline and single crystal diamond films, Chapter in: Carbon Based Nanomaterials, A. Öchsner, W. Ahmed, Eds. (Trans Tech, Switzerland, 2007), in press.

9. E. Kohn, P. Gluche, M. Adamschik, Diamond Relat. Maters. 8, (1999) 934.

10. F.J.H. Guillen, K. Janischowsky, J. Kusterer, W. Ebert, E. Kohn, Diamond Relat. Maters. 14, (2005) 411. 
11. D.M. Gruen, O.A. Shenderova, A.Ya. Vul' (Eds.), Synthesis, Properties and Applications of Ultrananocrystalline Diamond, Springer, 2005 (NATO Science Series part II, vol. 192).

12. P.W. May and M. Hannaway, Mat. Res. Symp. Proc. PV-956 (2006) 0956-J09-31.

13. S. Prawer, R.J. Nemanich, Phil. Trans. R. Soc. Lond. A 362 (2004) 2537.

14. J.W. Ager III, W. Walukiewicz, M. McCluskey, M.A. Plano, M.I. Landstrass, Appl. Phys. Lett. 66, (1995) 616.

15. F. Pruvost, E. Bustarret, A. Deneuville, Diamond Relat. Mater. 9, (2000) 295.

16. Y.G. Wang, S.P. Lau, B.K. Tay, X.H. Zhang, J. Appl. Phys., 92 (2002) 7253.

17. E. Gheeraert, P. Gonon, A. Deneuville, L. Abello, G. Lucazeau, Diamond Relat. Mater. 2 (1993) 742.

18. P. Gonon, E. Gheeraert, A. Deneuville, L. Abello, G. Lucazeau, J. Appl. Phys. 78 (1995) 7059.

19. R. Locher, J. Wagner, F. Fuchs, M. Maier, P. Gonon, P. Koidl, Diamond Relat. Mater. 4 (1995) 678.

20. M. Bernard, A. Deneuville P. Muret, Diamond Relat. Mater. 13, (2004), 282.

21. M. Bernard, C. Baron, A. Deneuville Diamond Relat. Mater. 13, (2004), 896.

22. J.P. Goss and P.R. Briddon, Phys. Rev. B 73, (2006) 085204.

23. D.W. Comerford, A. Cheesman, T.P.F. Carpenter, D.M.E. Davies, N.A. Fox, R.S. Sage, J.A. Smith, M.N.R. Ashfold, Yu. A. Mankelevich, J. Phys. Chem. A 110, (2006) 2868.

24. P.W. May, W.J Ludlow, M. Hannaway, J.A. Smith, K.N. Rosser, P.J. Heard, Chem. Phys. Letts. 446 (2007) 103.

25. P.W. May, W.J Ludlow, M. Hannaway, J.A. Smith, K.N. Rosser, P.J. Heard, submitted to Diamond Relat. Mater. (2007). 